\title{
Geometry, Mechanics, and Electronics of Singular Structures and Wrinkles in Graphene
}

\section{Citation}

Pereira, Vitor M., A. H. Castro Neto, H. Y. Liang, and L. Mahadevan. 2010. “Geometry, Mechanics, and Electronics of Singular Structures and Wrinkles in Graphene." Physical Review Letters 105 (15). https://doi.org/10.1103/physrevlett.105.156603.

\section{Permanent link}

http://nrs.harvard.edu/urn-3:HUL.InstRepos:41412115

\section{Terms of Use}

This article was downloaded from Harvard University's DASH repository, and is made available under the terms and conditions applicable to Open Access Policy Articles, as set forth at http:// nrs.harvard.edu/urn-3:HUL.InstRepos:dash.current.terms-of-use\#OAP

\section{Share Your Story}

The Harvard community has made this article openly available.

Please share how this access benefits you. Submit a story.

Accessibility 


\title{
Geometry, mechanics and electronics of singular structures and wrinkles in graphene
}

\author{
Vitor M. Pereira* and A. H. Castro Neto \\ Department of Physics, Boston University, 590 Commonwealth Avenue, Boston, MA 02215, USA \\ H. Y. Liang and L. Mahadevan ${ }^{\dagger}$ \\ School of Engineering and Applied Sciences, Harvard University, \\ 29 Oxford Street, Cambridge, Massachusetts 02138, USA
}

(Dated: November 12, 2018)

\begin{abstract}
As the thinnest atomic membrane, graphene presents an opportunity to combine geometry, elasticity and electronics at the limits of their validity. The availability of reliable atomistic potentials for graphene allows unprecedented precise simulations of the mechanical response of atomic membranes. Here we describe the transport and electronic structure in the neighbourhood of conical singularities, the elementary excitations of the ubiquitous wrinkled and crumpled graphene that occur in non-epitaxial suspended samples where shear stresses are unavoidable. We use a combination of atomistic mechanical simulations, analytical geometry and transport calculations in curved graphene, and exact diagonalization of the electronic spectrum to calculate the effects of geometry on electronic structure, transport and mobility in suspended samples. We also point out how the geometry-generated pseudo-magnetic/electric fields might disrupt Landau quantization under a magnetic field.
\end{abstract}

Graphene wrinkles easily and often [1]. This effect is most clearly observed in samples obtained from exfoliation of graphite, and subsequent deposition onto a substrate [2], or in chemically derived oxides [3]. Since graphene is an atomically thin membrane, it is impossible to lay a shear-free sheet of it onto a flat surface, as it sticks almost immediately to a substrate - such as the edges of a trench via van der Waals interaction - , and the substrate is itself rarely, if ever, flat [4-6], so that perfect shear-free conformations are not possible. In addition, recently advanced techniques to grow graphene on metallic surfaces clearly show widespread wrinkling arising from thermal expansion mismatch between graphene and the host substrate $[7,8]$. These boundary deformations acting on graphene lead to wrinkling because of the nearly negligible threshold for $1 \mathrm{D}$ and $2 \mathrm{D}$ buckling instabilities in thin plates; the bending rigidity scales with the cube of the thickness so that a thin membrane cannot support even arbitrarily small shear or compression without wrinkling on scales large compared to its thickness [9].

However, for all its flexural limpness, graphene exhibits the largest in-plane Young's modulus [10] and, though easy to bend, is extremely hard to stretch. This geometry-induced separation of the energy scales for thin membranes implies that they try to respond to shear by bending isometrically almost everywhere [11]. However, except in very limited cases corresponding to developable deformations, bending alone cannot accommodate the state of stress or the boundary conditions imposed by the geometry. This conflict is resolved naturally by local membrane stretching by an amount sufficient to just

\footnotetext{
* Corresponding author. Author email: vpereira@bu.edu.

${ }^{\dagger}$ Corresponding author. Author email: Im@seas.harvard.edu.
}

accommodate the imposed geometric and physical constraints, so that regions of in-plane strain are restricted to vanishingly small areas distributed throughout the system. A simple example is seen in a thin sheet of paper which is very resistant to stretching (it actually tears before we can stretch it), but bends easily; when a piece of crumpled paper is straightened out, we see flat areas connected by a network of ridges that meet at sharp vertices: the highly localized scars where the sheet is plastically deformed. The peaked structures constitute the basic element of the entire ridge network, and serve to focus large strains and energy densities. They are ubiquitous in thin films that are strongly deformed in such instances as drapes [11], skin winkles, etc., and are termed developable cones or conical singularities (CSs); their outer form has been geometrically and mechanically characterized in terms of a theory for the inextensional deformations of thin sheets Ref. [12, 13]. In particular, there is a simple universal analytic expression for their geometry as a function of the boundary and/or stress conditions on the sheet far from the nearly singular tip where the effects of stretching are concentrated.

Exfoliated graphene and suspended samples [2] naturally exhibit CSs as shown in Fig. 1(a), which are of particular interest in the quest for ultimate electronic mobility $[14,15]$ and non-trivial interaction effects $[16,17]$. Here we build on our knowledge of the structure and mechanics of CSs to study the influence of these ubiquitous objects on electronic transport in graphene. Unlike in most solid-state materials, flexural and planar deformations couple to electrons in graphene in a peculiar way. The honeycomb lattice implies that the effective electronic excitations of the system are two dimensional massless Dirac fermions [18]. The geometry of and strain in the lattice then couples to these excitations through both effective gauge fields, and local scattering potentials that follow the local curvature and thus affect 
the electronic structure $[19,20]$, opening the prospect for strain-engineered electronic devices [21, 22]. CSs are also present in buckled nanotubes, where they have been shown to significantly alter transport characteristics [23].

\section{CONICAL SINGULARITIES}

Graphene does behave like a thin plate under stress, even at the atomistic level; when sheared biaxially, and afterwards allowed to relax via Molecular Dynamics (MD) [24], Fig. 1(c) shows the relaxed configuration, which exhibits the classical Miura-ori like ridge pattern of 2D buckling [25], with the CSs arising at the intersection of the ridges Fig. 1(e,f).

In cylindrical coordinates, the displacement field associated with the CSs, a generalized cone, reads $\boldsymbol{u}(\rho, \theta)=$ $\boldsymbol{r}-\boldsymbol{r}_{0}=u_{\rho}(\rho, \theta) \boldsymbol{u}_{\rho}+u_{\theta}(\rho, \theta) \boldsymbol{u}_{\theta}+\zeta(\rho, \theta) \boldsymbol{z}$, where $\zeta(\rho, \theta)=$ $\rho \psi(\theta)$. The solution for $u_{\theta}(\rho, \theta), u_{\rho}(\rho, \theta)$, and $\psi(\theta)$ is obtained by solving the equations of equilibrium for the finite bending of a plate with the constraint of inextensibility, i.e. that there is no in-plane strain $\left(\gamma_{i j}=0\right)$. The vertical displacement is then given by (Refs. $[12,24]$ )

$$
\zeta=\rho \psi(\theta), \psi(\theta)=\varepsilon \Theta\left(|\theta|-\theta_{1}\right)+\varepsilon \Psi(\theta) \Theta\left(\theta_{1}-|\theta|\right) ;
$$

where $\varepsilon$ characterizes the angle of the enveloping cone, and both $\theta_{1} \approx 70^{\circ}$ and $\Psi(\theta)$ are universal. This independence of the shape on any material parameters and scale, together with the Cauchy-Born hypothesis, allows us to describe conical singularities and wrinkling in graphene by applying the deformation field $\boldsymbol{u}(\rho, \theta)$ to all atoms in the lattice. The resulting shape of the lattice is the one shown in Fig. 1(e), with the main effects arising from curvature. Since $\boldsymbol{u}(\rho, \theta)$ is constructed so that there is no in-plane strain; however some localized stretching strain is concentrated in the neighbourhood of the apex which will relax naturally in a MD simulation as a consequence of the large but finite stretching rigidity of graphene so that, even after relaxation, all inter-atomic distances are strongly peaked about the natural lattice spacing $a=a_{0}=1.42 \AA$, as can be seen in Fig. 1(d), with a spread of $2 \%$ for the values of $\varepsilon$ of interest here. This is just a reflection of the relative inextensibility of the in-plane $\sigma$ bonds, which leads to a blunting of the apex but is of little significance elsewhere. Since the relaxed structure shows strain $>1 \%$ for a dozen of atoms only, and very near the apex, we shall neglect it altogether.

\section{EFFECTIVE MODEL}

To understand how the electronic properties respond to this deformation, we note that the relevant physics occurs in the $p_{z}$-derived $\pi$ bands of graphene; curvature causes re-hybridization of these orbitals [26], hindering or favouring wavefunction overlap, and thus perturbs the electronic kinetic energy. This affects both the $\pi$ band sub-system and hybridizes the $p_{z}$ and the $s p^{2}$ sub-bands, which are otherwise orthogonal. As a first step we shall neglect this latter effect, which mostly shifts the chemical potential, and focus only on the $\pi$ bands.

Within the tight-binding approximation, the bandstructure is then determined by the effective Hamiltonian

$$
H=\sum_{\langle i, j\rangle} t_{i j} c_{i}^{\dagger} c_{j}+\sum_{\langle\langle i, j\rangle\rangle} t_{i j}^{\prime} c_{i}^{\dagger} c_{j}+\text { H. c. },
$$

where the two contributions come from first and second neighbours, and $t_{i j}^{(\prime)}=V_{p p \pi}$ is the two centre SlaterKoster overlap integral [27], which has to be calculated now for all pairs of neighbours, taking into consideration the full geometry of the deformed lattice. To do this, we introduce the unit normal at every point of the surface, $\boldsymbol{n}(\rho, \theta)$, so that for two atoms separated by an arbitrary distance $\boldsymbol{d}=\boldsymbol{R}_{i}-\boldsymbol{R}_{j}$, straightforward rotation of the $p_{z}$ orbitals and Slater-Koster tables tell us that the overlap integral is [28]

$$
t_{i j}=V_{p p \pi} \boldsymbol{n}_{i} \cdot \boldsymbol{n}_{j}+\left(V_{p p \sigma}-V_{p p \pi}\right)\left(\boldsymbol{n}_{i} \cdot \hat{\boldsymbol{d}}\right)\left(\boldsymbol{n}_{j} \cdot \hat{\boldsymbol{d}}\right) .
$$

Since the surface is completely parametrized by the normal displacement field, we may use the geometry of the developable cone to obtain the normals, distances and the hopping $t_{i j}$ among any two atoms, noting that the underlying metric remains Euclidean. To make progress analytically we assume, with Harrison [29], that $d^{2} V_{p p x}(d)=$ $d_{0}^{2} V_{p p x}\left(d_{0}\right)$, so that on solving the Gauss equations we obtain $t_{i j}(\boldsymbol{d})=t_{i j}^{0}\left(\boldsymbol{d}_{0}\right)+\delta t_{i j}$, with:

$$
\delta t_{i j} \approx-V_{p p \pi}^{0} \frac{1}{2}\left|\left(\boldsymbol{d}_{0} \cdot \boldsymbol{\nabla}\right) \boldsymbol{\nabla} \zeta\right|^{2}+\frac{V_{1}}{d_{0}^{2}}\left[\left(\boldsymbol{d}_{0} \cdot \boldsymbol{\nabla}\right)^{2} \zeta\right]^{2},
$$

$V_{1}=V_{p p \pi}^{0} / 3-V_{p p \sigma}^{0} / 4$. In the low energy approximation, we may then describe (2) by the effective Dirac Hamiltonian: $H \approx v_{F} \boldsymbol{\sigma} \cdot\left[\boldsymbol{p}-\frac{1}{v_{\mathrm{F}}} \mathcal{A}(\boldsymbol{r})\right]+\left[3 t_{0}^{\prime}+\Phi(\boldsymbol{r})\right] \sigma^{0}$ in each valley of the Brillouin zone. Then the effective gauge field $\mathcal{A}(\boldsymbol{r})$ and the local potential $\Phi(\boldsymbol{r})$ depend, respectively, on the perturbations of the nearest neighbour, and next-nearest neighbour hopping Ref. [18] via

$$
\mathcal{A}_{x}-i \mathcal{A}_{y}=\sum_{\boldsymbol{n}} \delta t_{\boldsymbol{n}}(\boldsymbol{r}) e^{i \boldsymbol{k} \cdot \boldsymbol{n}}, \Phi=\sum_{\Delta} \delta t_{\Delta}^{\prime}(\boldsymbol{r}) e^{i \boldsymbol{k} \cdot \boldsymbol{\Delta}} .
$$

Substituting (4) in (5), one obtains

$$
\Phi(\boldsymbol{r})=\alpha \operatorname{Tr}^{2}\left[\partial^{i} \partial_{j} \zeta\right]-\beta \operatorname{det}\left[\partial^{i} \partial_{j} \zeta\right]
$$

with $\alpha=9 a_{0}^{2} V_{p p \pi}^{0} / 8+27 a_{0}^{2} V_{p p \sigma} / 32 \approx 1.5 \mathrm{eV} \AA^{2}$, and $\beta=3 a_{0}^{2} V_{p p \pi}^{0}+9 a_{0}^{2} V_{p p \sigma}^{0} / 8 \approx 3 \mathrm{eV} \AA^{2}$ [30]. We recall that $\partial^{i} \partial_{j} \zeta \approx K^{i}{ }_{j}$ is the curvature tensor of our conical surface and, since $H=\operatorname{Tr} K^{i}{ }_{j} / 2$ and $\operatorname{det} K^{i}{ }_{j}$ are the local mean and Gaussian curvatures, it follows that $\Phi$ is entirely determined by the cone geometry. Moreover, since CSs are developable surfaces, the Gaussian curvature vanishes everywhere, so that $\Phi(\boldsymbol{r})=\alpha a_{0}^{2}\left(\nabla^{2} \zeta\right)^{2}$. The gauge field 
$\mathcal{A}$ is also given in terms of products of $\partial^{i} \partial_{j} \zeta$, but we shall not write it explicitly since this potential couples to the electric current, and therefore does not contribute in leading order for scattering and transport when timereversal symmetry is preserved. However $\Phi$ leads to an electrostatic potential that is felt by the Dirac electrons and thus contributes directly to the resistivity.

\section{TRANSPORT}

We now consider the contribution of the CSs for the momentum relaxation time in the Boltzmann formalism. In the Born approximation, the scattering rate is given by $S\left(\boldsymbol{k}, \boldsymbol{k}^{\prime}\right)=2 \pi / \hbar\left|V_{\boldsymbol{k}, \boldsymbol{k}^{\prime}}\right|^{2} \delta\left(E_{\boldsymbol{k}}-E_{\boldsymbol{k}^{\prime}}\right)$, with $V_{\boldsymbol{k}, \boldsymbol{k}^{\prime}}=\Phi_{\boldsymbol{k}-\boldsymbol{k}^{\prime}}\left[1+\exp \left(i \phi_{\boldsymbol{k}}-i \phi_{\boldsymbol{k}^{\prime}}\right)\right] / 2$, and $\Phi_{\boldsymbol{q}}$ is the Fourier transform of the local potential (6): $\Phi(\boldsymbol{r})=$ $\alpha a_{0}^{2}\left[\psi(\theta)+\psi^{\prime \prime}(\theta)\right] / r^{2}$, which is of course directly related to the cone geometry. This potential is unusual for two reasons: it is anisotropic on account of (1) and decays in space as $\propto 1 / r^{2}$, so that it is beyond the supercritical threshold for Dirac fermions in 2D [31]. Were it not for the natural lattice regularization at $r \sim 0$, such potential would lead to an unbound spectrum of discrete states. This effect is also blunted the mechanical, stretchinduced relaxation observed near the apex in Fig. 1(e,f). The result is a short range potential with a finite number of bound states (unlike the Coulomb case where the long range $1 / r$ tail begets an infinite spectrum of resonances, even after regularization), so that CSs therefore scatter as short range, anisotropic potentials.

The $1 / r^{2}$ decay in the potential leads to an infrared divergence in $\Phi_{\boldsymbol{q}}$ with a leading order isotropic contribution $\Phi_{\boldsymbol{q}} \approx-10 \alpha \varepsilon^{2} \log \left(q r_{0}\right)$, all anisotropy being hidden in the subleading terms, with the regularization distance, $r_{0}$ of the order of the lattice spacing, reflecting the relaxation in the neighbourhood of the apex. Then, the CSs scatter primarily as an isotropic $1 / r^{2}$ potential, and the scattering time for the potential $\nu_{0} / r^{2}$ can be calculated exactly, and reads

$$
\frac{1}{\tau\left(k_{F}\right)}=\frac{2 \pi^{2} n_{i} \nu_{0}^{2}}{v_{\mathrm{F}} \hbar^{2}} k_{F} G_{2,4}^{3,1}\left(\begin{array}{l|l}
4 k_{F}^{2} r_{0}^{2} & \begin{array}{c}
-\frac{1}{2}, \frac{1}{2} \\
0,0,0,-2
\end{array}
\end{array}\right),
$$

where $G$ is a Meijer function [32], $n_{i}$ the density of scatterers, and $k_{F}$ relates to the carrier density via $k_{F}^{2}=\pi n_{e}$. Then the longitudinal conductivity follows from Eq. (7) and yields

$$
\sigma=\sigma_{0} G_{2,4}^{3,1}\left(4 k_{F}^{2} r_{0}^{2} \mid \begin{array}{c}
-\frac{1}{2}, \frac{1}{2} \\
0,0,0,-2
\end{array}\right)^{-1}, \quad \sigma_{0}=\frac{v_{\mathrm{F}}{ }^{2} \hbar e^{2}}{2 \pi^{3} n_{i} v_{0}^{2}}
$$

which is only relevant in the regime $0<k r_{0} \lesssim 1$ shown in Fig. 2. We see that the conductivity is essentially linear in electron density throughout most of the region of interest, except for the logarithmic singularity around the Dirac point, where it grows sub-linearly. The corresponding approximate mobility is $\mu \approx \frac{6 v_{F}^{2} \hbar e r_{0}^{2}}{\pi^{2} v_{0}^{2}} \frac{1}{n_{i}}$, and when $\nu_{0}$ is replaced by the corresponding parameter for $\mathrm{CSs}\left(\nu_{0} \rightarrow 67 \alpha \varepsilon^{2} / \pi \mathrm{eV} \AA^{2}\right)$ one obtains the mobility for a sea of uncorrelated CSs as $\mu \approx 10^{29} r_{0}^{2} /\left(n_{i} \varepsilon^{4}\right) \mathrm{cm}^{2} /(\mathrm{Vs})$. Substituting the parameter values $r_{0} \sim 5 \AA$ and $n_{i} \sim$ $10^{12} \mathrm{~cm}^{-2}$, results in $\mu \sim 10^{3} / \varepsilon^{4} \mathrm{~cm}^{2} /(\mathrm{Vs})$. The $\varepsilon^{4}$ dependence reflects a strong sensitivity to the aperture of the enveloping cone of each CS, but given that $\varepsilon \lesssim 0.5$, it causes relatively small scattering. This effect should thus be more important in high- mobility suspended samples, where the CSs can become a limiting factor in carrier mobility.

\section{ELECTRONIC SPECTRUM}

Although the gauge fields $\mathcal{A}$ are not expected to contribute to transport at leading order, they do influence the electronic spectrum. In fact, since they arise from perturbations to nearest neighbour hopping, they might cause considerable fictitious magnetic fields [22]. To address this at the level of the lattice, we have calculated the electronic structure associated with the full tightbinding Hamiltonian (2) in the presence of a single unrelaxed CS. The local density of states [LDOS, $N_{\boldsymbol{r}}(E)$ ] for representative parameters is shown in Fig. 3. We see that CSs scatter strongly enough to create bound electronic states as shown in Fig. 3(a,c) by the sharp peaks for states beyond the band edge, decaying rapidly away from the apex. In addition, the LDOS is very structured at other energies within the band, signalling the formation of resonant states. This is more clearly visible in Fig. 3(c) where the sampling points lie in the region of higher curvature. In this case the LDOS curves show even stronger perturbation around the Dirac point. The local bandwidth is decreased, and the leading slope of $N_{r}(E)$ around $E \approx 0$ fluctuates, indicating renormalized Fermi velocities in the neighbourhood of the apex. In panels Fig. 3(d-e) we plot the real-space distribution of the LDOS at representative energies around the Dirac point, showing that the charge density is mostly localized in the apex, albeit with a "leak" along two rays that are at an angle $\approx 24^{\circ}$ with the axis of symmetry of the CSs, coinciding with the two zero curvature generators in the entire conical surface (1), and show clearly the role of curvature-induced confinement [24]. We also always see signals of "magnetic" oscillations around the Dirac point, as shown in Fig. 3 (a)[33]. Even though these studies are carried out with zero magnetic field, the presence of these locally varying fictitious fields is expected to influence Landau level quantization under a real magnetic field.

\section{DISCUSSION}

We have shown that CSs have potential to markedly affect electronic properties and transport in wrinkled graphene. They contribute a quasi linear-in-density conductivity, and might even limit mobility in suspended 
samples at low temperatures, even in the dilute limit. We did not consider the anisotropy in the transport calculations, but it is likely to play a role in situations like Fig. 1(b), where a strong alignment might lead to coherent scattering. In suspended samples, curvature-induced disordered flux might be dominant and thus explain why the quantum Hall effect in 4-terminal suspended samples is so elusive. Our calculations suggest that it is possible to engineer the electronic and transport properties in graphene by inducing and controlling CSs on demand using substrate shear, or by exploring the anomalous thermal expansion coefficient of graphene, as initiated in Ref. [2]. Finally, such strong impact of singular deformations on the electronic system can pave new avenues of interplay between structure and electronics. Graphene, as seen, has clear and unprecedented advantages, insofar as both its mechanical response and electronic structure are easily and accurately modelled down to the atomic level.

\section{Acknowledgments}

AHCN acknowledges the partial support of the U.S. DOE under grant DE-FG02-08ER46512, and ONR grant MURI N00014-09-1-1063. HL and LM acknowledge the support of the Harvard-NSF MRSEC and LM acknowledges support from the MacArthur Foundation.
[1] Jannik C. Meyer et al., Nature 446, 60 (2007).

[2] W. Bao et al., Nature Nano. 4, 562 (2009).

[3] S. Stankovich et al., Carbon 45, 1558 (2007).

[4] E. Stolyarova et al., Proc. Natl. Acad. Sci. USA 104, 9209 (2007).

[5] M. Ishigami et al., Nano Lett. 7, 1643 (2007).

[6] V. Geringer et al., Phys. Rev. Lett. 102, 076102 (2009).

[7] S. Bae, H. K. Kim, X. Xu, J. Balakrishnan, T. Lei, Y. I. Song, Y. J. Kim, B. Ozyilmaz, J.-H. Ahn, B. H. Hong, et al. (2009), 0912.5485.

[8] P. Sutter, J. T. Sadowski, and E. Sutter, Phys. Rev. B 80, 245411 (2009).

[9] L. D. Landau and E. M. Lifshitz, Theory of Elasticity (Pergamon, 1986), 3rd ed.

[10] C. Lee et al., Science 321, 385 (2008).

[11] E. Cerda et al., Proc. Natl. Acad. Sci. 101, 1806 (2004).

[12] E. Cerda and L. Mahadevan, Phys. Rev. Lett. 80, 2358 (1998).

[13] E. Cerda and L. Mahadevan, Proc. R. Soc. A 461, 671 (2005).

[14] K. Bolotin et al., Solid State Comm. 146, 351 (2008).

[15] G. Li, A. Luican, and E. Y. Andrei, Phys. Rev. Lett. 102, 176804 (2009).

[16] X. Du et al., Nature 462, 192 (2009).

[17] K. Bolotin et al., Nature 462, 196 (2009).

[18] A. H. Castro Neto et al., Rev. Mod. Phys. 81, 109 (2009).

[19] Vitor M. Pereira et al., Phys. Rev. B 80, 045401 (2009).

[20] R. M. Ribeiro et al., New J. Phys. 11, 115002 (2009).

[21] V. M. Pereira and A. H. Castro Neto, Phys. Rev. Lett. 103, 046801 (2009).

[22] F. Guinea, M. I. Katsnelson, and A. K. Geim, Nature Phys. 6, 30 (2009).

[23] M. R. Falvo et al., Nature 389, 582 (1997); D. Bozovic et al., App. Phys. Lett. 78, 3693 (2001) .

[24] Accompanying supplementary information.

[25] L. Mahadevan and S. Rica, Science 307, 1740 (2005).

[26] E.-A. Kim and A. H. C. Neto, Europhys. Lett. 84, 57007 (2008).

[27] J. C. Slater and G. F. Koster, Phys. Rev. 94, 1498 (1954).

[28] A. Isacsson et al., Phys. Rev. B 77, 035423 (2008).

[29] W. A. Harrison, Elementary Electronic Structure (World Scientific, 1999).

[30] In our calculation we use $V_{p p \pi}\left(a_{0}\right)=3 \mathrm{eV}, V_{p p \sigma}\left(a_{0}\right)=$
$1.7 V_{p p \pi}\left(a_{0}\right), V_{p p \pi}\left(\sqrt{3} a_{0}\right)=0.1 V_{p p \pi}\left(a_{0}\right), V_{p p \sigma}\left(\sqrt{3} a_{0}\right)=$ $0.1 V_{p p \sigma}\left(a_{0}\right)$.

[31] D. Novikov, Phys. Rev. B 76, 245435 (2007).

[32] I. S. Gradshteyn and I. M. Ryzhik, Table of Integrals, Series, and Products (World Scientific, 1965), 4th ed.

[33] The unrelaxed surface has no in-plain strain, but the 3D interatomic distances in the curved lattice do change.

[34] Images courtesy of A.K. Geim and C.N. Lau, respectively.

[35] Donald W Brenner et al., J. Phys. C 14, 783 (2002). 

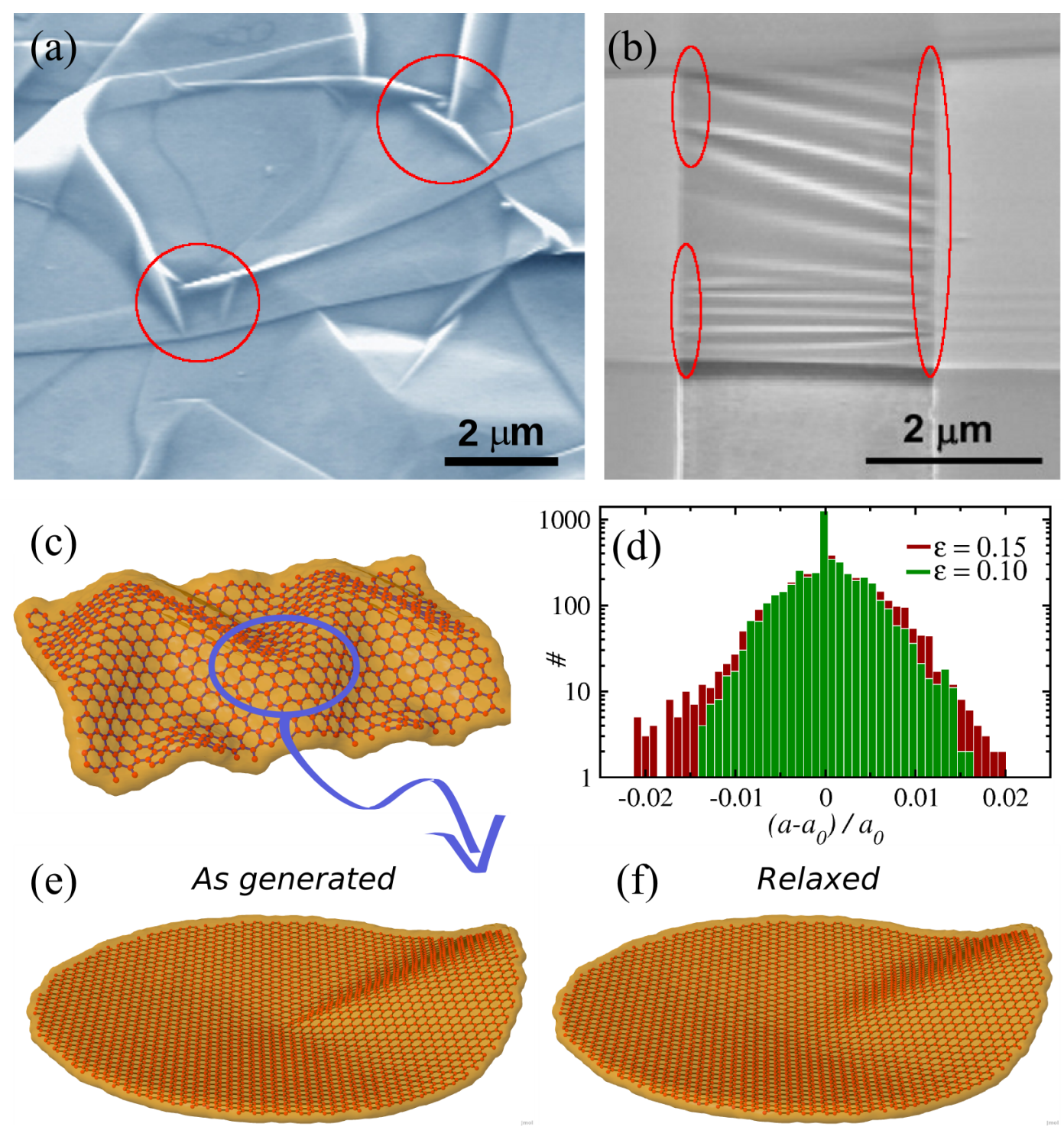

Figure 1: Wrinkles in graphene and the origin of conical singularities. a, Folded graphene sheet resembling the draping of a textile, and graphene suspended over a trench, b [34]. Some regions with visible conical singularities (CSs) are highlighted. c, Relaxed atomistic profile of a portion of graphene under biaxial shear, displaying typical buckling ridges. d, Logarithmic histogram of the inter-atomic distances in the relaxed configuration $\mathbf{f}$ for two values of $\varepsilon$. e,f, Profile of the CSs studied here $(\varepsilon=0.1)$. The atomic positions are shown as generated by applying $\boldsymbol{u}(\rho, \theta)$ to all atoms, e, and after relaxation by MD, $\mathbf{f}$. 


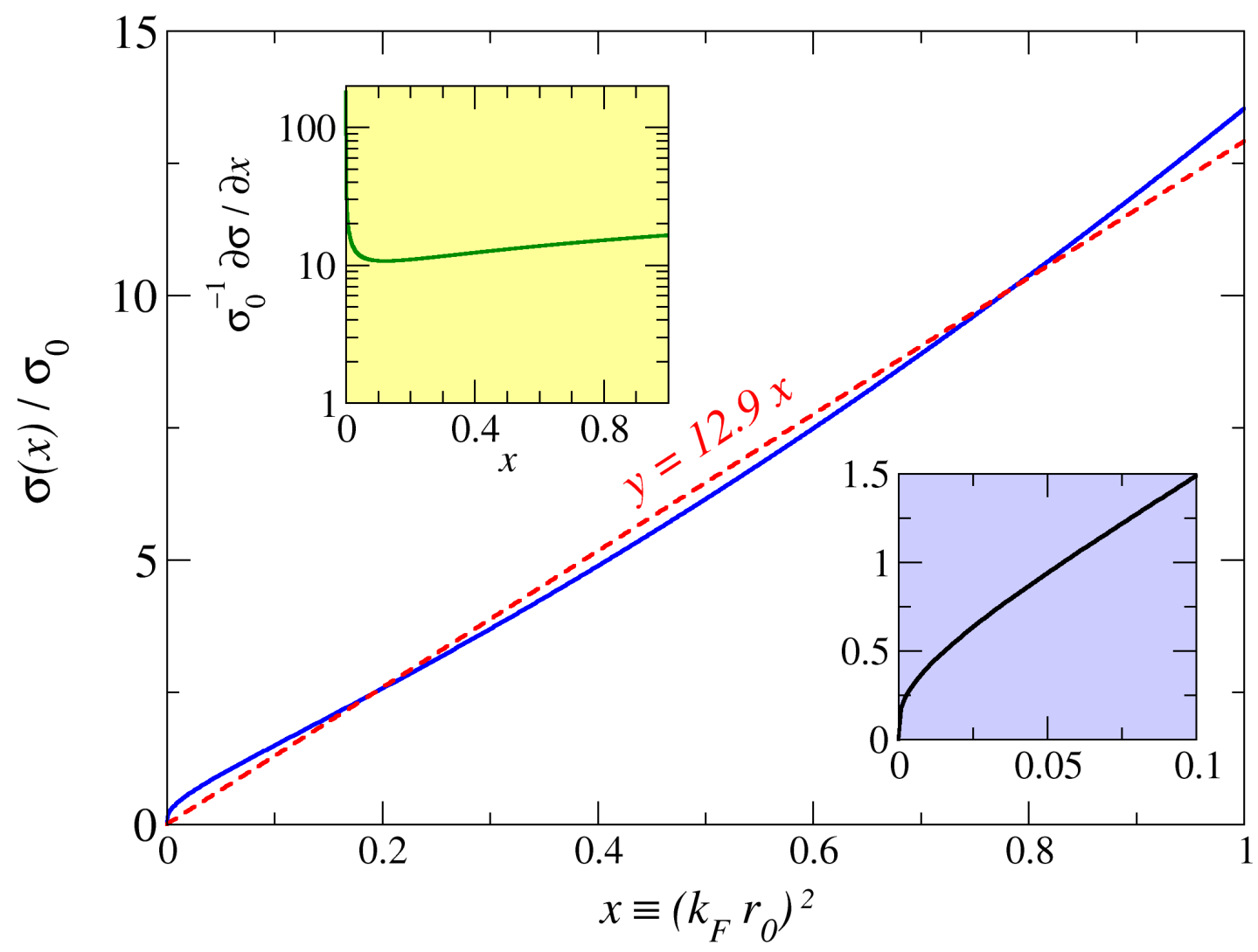

Figure 2: DC conductivity under the influence of CS's. DC conductivity (8) versus the adimensional electron density $x \equiv\left(k_{F} r_{0}\right)^{2}$. The dashed line shows the best linear fit in the entire domain $(y \simeq 12.9 x)$. Top inset shows $\sigma_{0}^{-1} \partial \sigma / \partial x$, which is how the electronic mobility $(\mu)$ is frequently extracted experimentally. Bottom inset amplifies the region $k_{F} \sim 0$, dominated by a log singularity. 

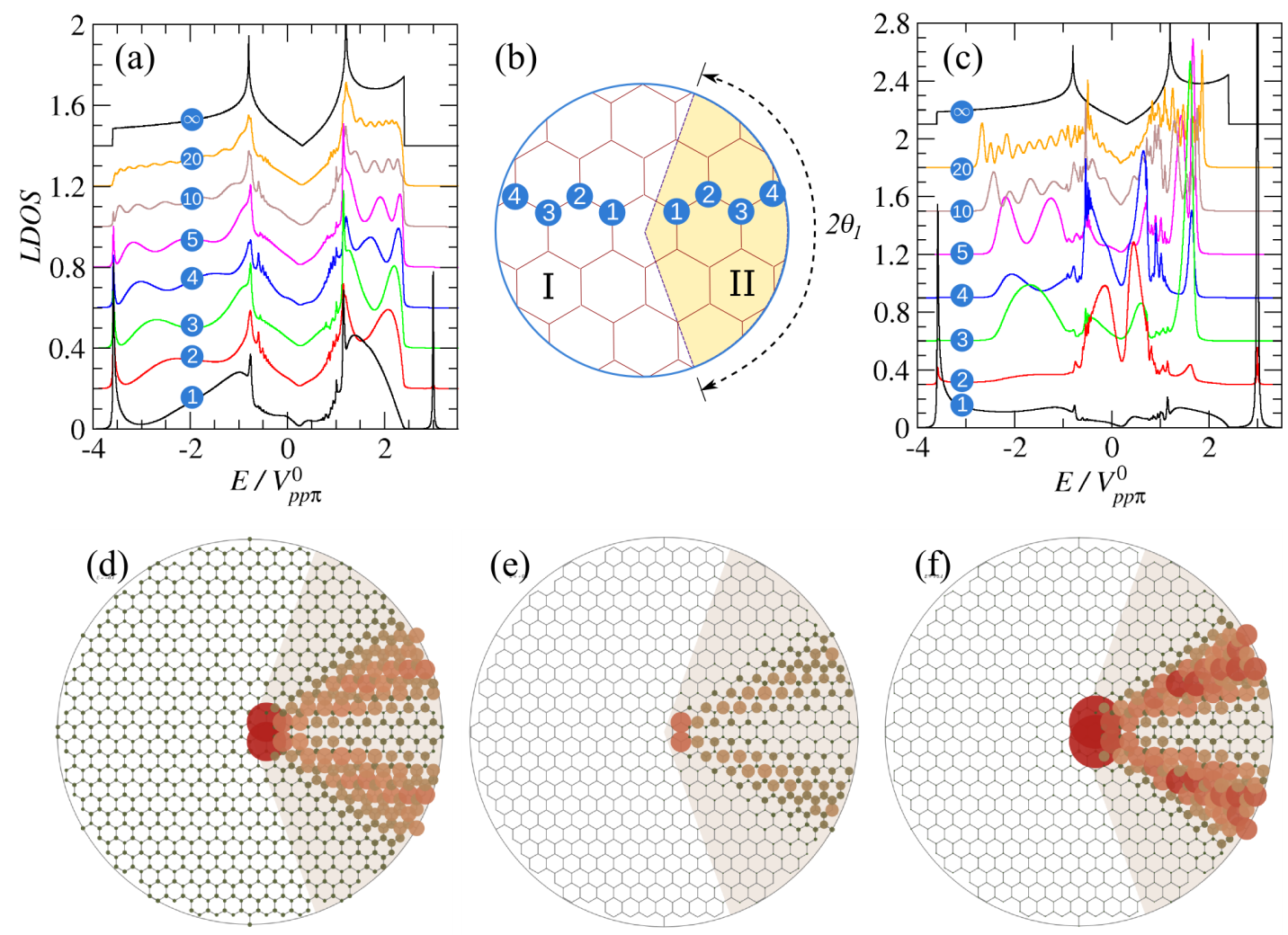

Figure 3: LDOS around the apex of a CS. LDOS close to the apex of a CS with $\varepsilon=0.3$, in the regions I, a, and II, c, and at specific lattice positions, as specified in panel b. The curves in a,c were vertically shifted for clarity. In d-f we show the LDOS in real space for selected energies: $E / V_{p p \pi}^{0}=-0.1,0.3,0.4$. More detailed plots are available in [24]. 


\section{Supplementary Material}

\section{Parametrization of conical singularities}

The fundamental object associated with a crumpled or wrinkled graphene sheet is the conical singularity [12], for which there is an analytic solution that minimizes the elastic energy subject to the constraint of inextensional deformations. In cylindrical polar coordinates $(\rho, \theta)$, the displacement field relative to the flat state is given by $\boldsymbol{u}(\rho, \theta)=\boldsymbol{r}-\boldsymbol{r}_{0}=u_{\rho}(\rho, \theta) \boldsymbol{u}_{\rho}+u_{\theta}(\rho, \theta) \boldsymbol{u}_{\theta}+\zeta(\rho, \theta) \boldsymbol{z}$, where the vertical displacement $\zeta(\rho, \theta)=\rho \psi(\theta)$ is a generalized cone. The azimuthal component of the displacement field $\psi(\theta)$ is given by

$$
\psi(\theta)=\varepsilon \Theta\left(|\theta|-\theta_{1}\right)+\varepsilon \Psi(\theta) \Theta\left(\theta_{1}-|\theta|\right) .
$$

where $\Theta(\cdot)$ is the usual Heaviside function, the aperture angle of the enveloping cone is $\pi-2 \tan ^{-1} \varepsilon$, and the universal geometric function $\Psi(\theta)$ is given by:

$$
\Psi(\theta)=\frac{\sin \theta_{1} \cos a \theta-a \sin a \theta_{1} \cos \theta}{\sin \theta_{1} \cos a \theta_{1}-a \sin a \theta_{1} \cos \theta_{1}},
$$

where $a \approx 3.8$ and $\theta_{1} \approx 70^{\circ}$ are universal numbers. The general shape of the deformed surface is shown in Fig. 4(a). The domain $|\theta|<\theta_{1}$ corresponds to the region where the surface coincides with the cylindrical envelope cone.

The local azimuthal curvature of the cone is $\left[\psi^{\prime \prime}(\theta)+\right.$ $\psi(\theta)] / \rho$. In Fig. 4(b) we present a contour plot of $\varepsilon\left[\psi^{\prime \prime}(\theta)+\psi(\theta)\right]$. For the region $|\theta|>\theta_{1}$, the shape is not that of a perfect cone; instead the local mean curvature increases attaining a maximum at $\theta_{0} \approx 47^{\circ}$, before decreasing with an inflection point at $\theta_{2} \approx 24^{\circ}$ and becomes negative for $|\theta|<\theta_{2}$, with a maximum at $\theta=0$.

\section{Details of the atomistic calculations}

We start with a graphene disk of radius $\sim 5 \mathrm{~nm}$ which is geometrically transformed into a conical shape following the inextensional analytic model of [12]. This is done by fixing the coordinates of the atoms of 2 rows near the free edge, while the remaining carbon atoms of the cone are allowed to relax via a classical molecular dynamics simulation at zero temperature, so that the analytic solution which is singular at the tip relaxes by stretching locally. The $\mathrm{C}-\mathrm{C}$ interactions are modeled with a second generation reactive empirical bond order (REBO) potential [35].

\section{Details of the LDOS calculations}

We use a $\pi$ tight-binding Hamiltonian (2), including overlaps up to next-to-nearest neighbors on a real Honeycomb lattice of dimensions $2000 \times 1000 a_{0}^{2}$. The lattice was deformed to conform to the exact analytical profile of the conical indentation discussed in the main text. The apex of the cone lies at the center of the lattice. The hopping integrals $t_{i j}$ and $t_{i j}^{\prime}$ were determined from the analytical result (3). The local density of states (LDOS) was calculated by recursivelly solving for the local Green's function, until convergence is achieved. The results for the LDOS, $N_{\boldsymbol{r}}(E)$, are hence numerically exact, up to a broadening of $\Delta E / V_{p p \pi}^{0}=0.01$, employed for faster convergence.

\section{Real space distribution of LDOS}

The LDOS, $N_{\boldsymbol{r}}(E)$, has been calculated exactly, as discussed above, at all lattice sites in the vicinity of the apex. for readability, the real-space images show only a portion - less than $1 \%$ in area - of the total lattice. Since our interest rest on the local electronic structure near the apex, we considered only one conical singularity. The large lattice size ensures that spurious edge effects do not interfere with the structure around the apex.

In Fig. 5 we present $N_{\boldsymbol{r}}(E)$ at all those lattice positions, $\boldsymbol{r}$, for a constant energy, $E$. The energy is measured in units of $V_{p p \pi}^{0}$. At each lattice site a disk, whose diameter is proportional to the magnitude of the LDOS, is drawn. The panels in Fig. 5 pertain to an interval of energies between $E / V_{p p \pi}^{0}=-1.7$ and $E / V_{p p \pi}^{0}=2.1$, at increments of 0.2 . This interval encompasses the two van Hove singularities, and the Dirac point which, for our choice of parameters, lies at $E / V_{p p \pi}^{0}=0.3$.

For comparison, in Fig. 6 we present precisely the same analysis for a wider cone, with $\varepsilon=0.1$. In this case, there are no visible features, with the LDOS remaining greatly homogeneous, and following a variation with energy much similar to the unperturbed lattice. Fig. 7 shows an exaggerated case with $\varepsilon=0.5$. In this case relaxation effects should be stronger and the approximation of neglecting $\sigma-\pi$ overlaps less warranted. Data is shown here for perspective only. 
(a)

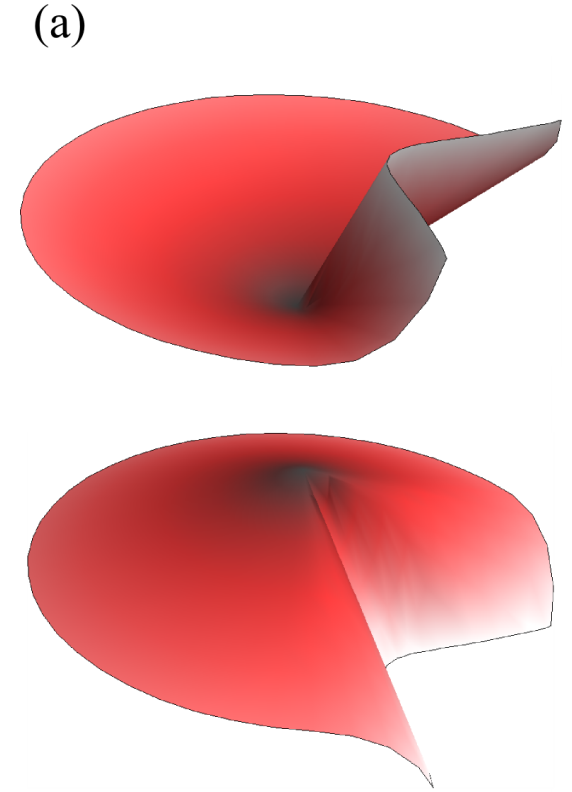

(b)

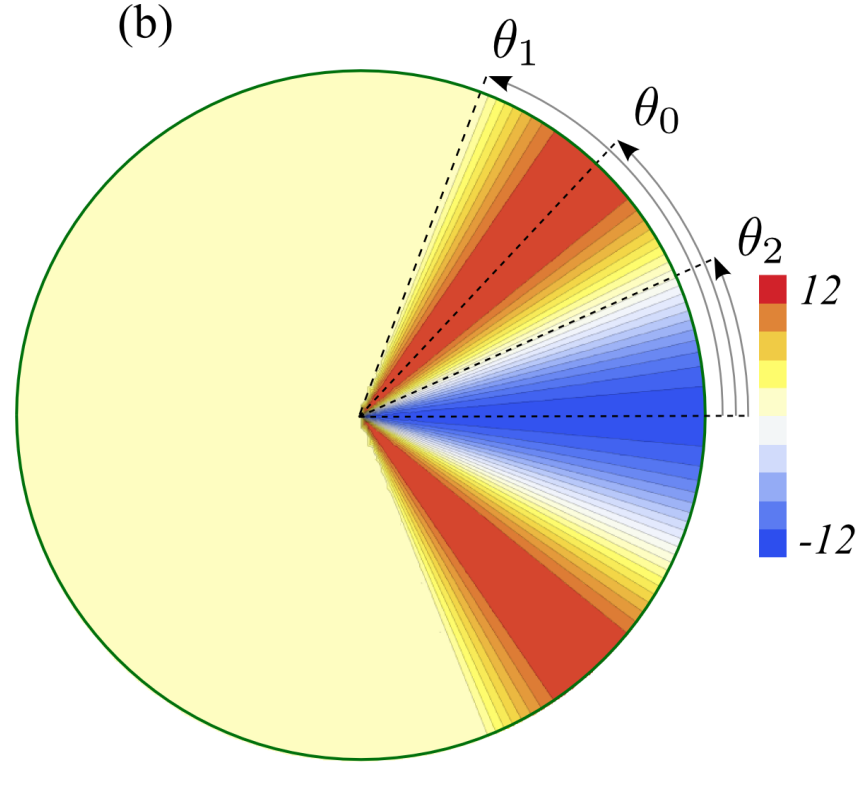

Figure 4: Geometry of a conical singularity. a, profile of a conical singularity with $\varepsilon=0.2$ seen from two different perspectives. b. Contour plot of $\varepsilon\left[\psi^{\prime \prime}(\theta)+\psi(\theta)\right]$ revealing the curvature as a function of $\theta$. The angles $\theta_{1} \approx 70^{\circ}, \theta_{0} \approx 46^{\circ}$, and $\theta_{2} \approx 24^{\circ}$ denote the generators along which the surface detaches from the enveloping cone, along which the curvature is maximal, and along which the curvature is null, respectively. 

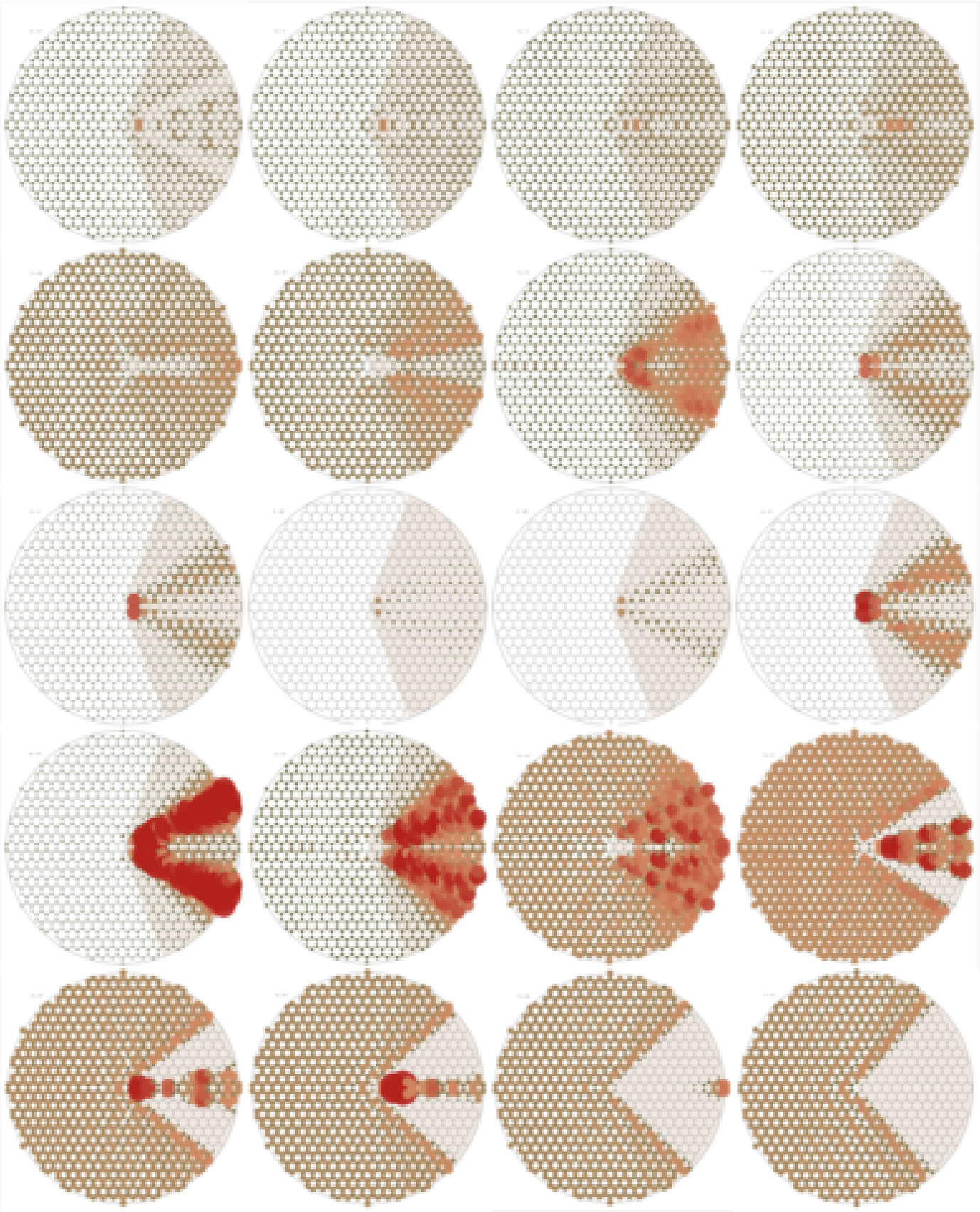

Figure 5: LDOS around the apex af a conical singularity. Each panel shows the distribution of the LDOS in real space around the apex of a cone with $\varepsilon=0.3$. Panels pertain to energies $E / V_{p p \pi}^{0}=-1.7,-1.5,-1.3, \ldots, 1.9,2.1$. 



Figure 6: LDOS around the apex af a conical singularity. Each panel shows the distribution of the LDOS in real space around the apex of a cone with $\varepsilon=0.1$. Panels pertain to energies $E / V_{p p \pi}^{0}=-1.7,-1.5,-1.3, \ldots, 1.9,2.1$. 


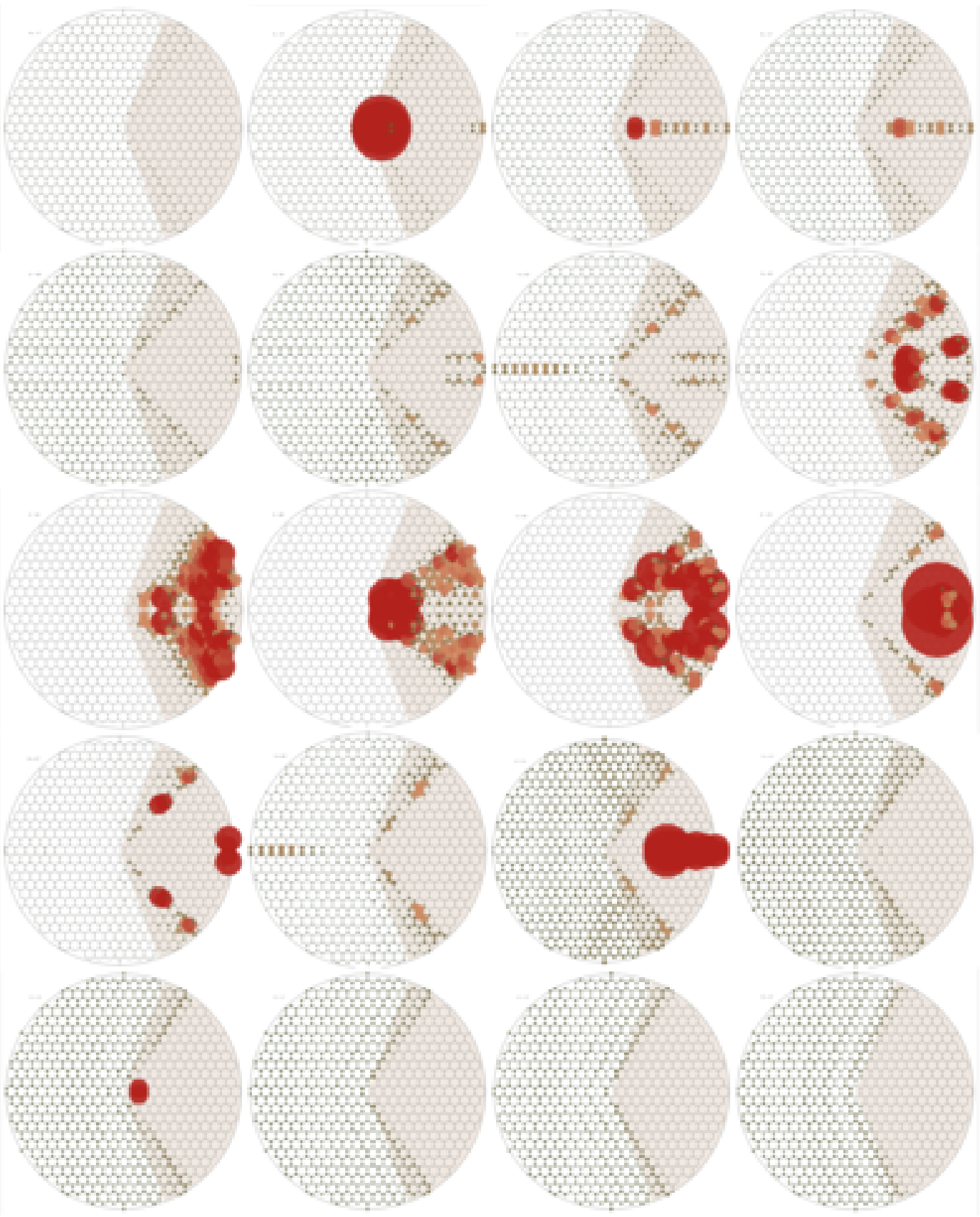

Figure 7: LDOS around the apex af a conical singularity. Each panel shows the distribution of the LDOS in real space around the apex of a cone with $\varepsilon=0.5$. Panels pertain to energies $E / V_{p p \pi}^{0}=-1.7,-1.5,-1.3, \ldots, 1.9,2.1$. 